\title{
Smart Electric Vehicle Charging Through Cloud Monitoring and Management
}

\author{
Mohd. Saqib ${ }^{1}$ (D) Md. Muzakkir Hussain ${ }^{2}$ - Mohammad Saad Alam ${ }^{3}$. \\ M. M. Sufyan Beg ${ }^{2}$. Amol Sawant ${ }^{4}$
}

Received: 27 April 2017 / Accepted: 21 September 2017 / Published online: 10 October 2017

(C) Springer Nature Singapore Pte Ltd. 2017

\begin{abstract}
Smart charging system of electric vehicle using cloud based monitoring and management is demonstrated in this work. xEVs (electric plugin hybrid, battery electric vehicles) Charging Management System is crucial for the dynamic demands of charging infrastructure, namely perspectives from automakers, electricity providers, vehicle owners and charging service providers. Through dedicated interface, the developed system is capable of providing real time information to $\mathrm{xEVs}$ users regarding nearest charging station with minimum queuing delay, with minimum charging cost through a secured online accessing mechanism for accessing Sate of the Charge (SOC) of the xEV's
\end{abstract}

Mohd. Saqib

Saqib9828@gmail.com

Md. Muzakkir Hussain

md.muzakkirhussain@zhcet.ac.in

Mohammad Saad Alam

Saad.alam@zhcet.ac.in

M. M. Sufyan Beg

mmsbeg@eecs.berkeley.edu

Amol Sawant

Amol.sawant@indismartgrid.org

1 Department of Computer Science, Aligarh Muslim University, Aligarh, India

2 Department of Computer Engineering, Aligarh Muslim University, Aligarh, India

3 Centre of Advanced Research in Electrified Transportation, Aligarh Muslim University, Aligarh, India

4 India Smart Grid Forum (ISGF), New Delhi, India battery being charged. The system not only provide an execution framework for the xEVs users but also provide an optimal energy trading solution to all entities involved in a smart charging infrastructure such as charging station, aggregators, smart grid etc. The work also explains the cloud enabled bidding strategies that look for day-ahead and term-ahead markets. The aggregators will use the smart decisions undertaken by cloud analytics to execute their bidding strategies in way to maximize the profit. Further, the work also assesses the possible cyber security aspects of such architectures along with providing possible solutions.

Keywords Electric Vehicles (EVs) - Cloud computing · Cloud management $\cdot$ Power market operation $\cdot$ Smart grid

\section{Introduction}

The rapid urbanization and industrialization are posing pollution issues as the prime concern in India [1]. Automotive sector constitute the main sources of such pollution. Air pollution is often understood as an urban issue [2] and it is appealing that cities concentrate economic activity and demand for energy services, and so tend to experience heavy concentrations of harmful air pollutants. Electric vehicles [3] potentially emerged as supreme player in realizing an eco-friendly, pollution free means of next generation transport [4]. Due to venturing features associated to electric vehicles, they are acquiring a consensus call from automotive industries, R\&Ds, policymakers contrast to their ICE counterparts for utility providers and industrial sector [5-7].

Heavy penetration of xEVs will create enormous demand sink for electrical energy needed to charge xEVs [8]. An un coordinated charging infrastructure will create sudden demand upsurges, put constraints on the underlying power 
grid and at the worst case may lead to failures and outages [9]. The demand of electrical power will be different at different charging station according to number of xEVs deployed or routed per station. However, all the charging stations require strict timing constraints in their charging patterns so that the XEVs could be charged serviced reliably [10]. As the charging stations are coupled to the mains supply system the power drawn by them in different time instants should not induce encumbrance on the back end smart grid (SG) system [11]. Thus, it is the call of the hour to develop a centralized EV/PHEV charging management system which controls demand and supply problem within the dedicated time frames.

Meanwhile, the xEVs, emerged as a new kind of power load, could perform active role in diverse energy market services such as vehicle to grid (V2G) [12], frequency regulation [13], ancillary services [14] etc, and would potentially exert an incredible impact on the daily residential load curve if they are properly managed [15]. But such abilities latent in the xEV fleet could only be brought to commercial use if their charging and discharging methodologies are intelligently tuned [16].

Thus there is a need for benchmarking efficient management protocols and standards to ensure that the fleets are regulated smartly [5].

Electric vehicles can act as the reliable and sustainable powertrain alternative for the future generation transport, its inclusion in the existing infrastructure can pose challenge for operation and maintenance activities unless they are intelligently deployed. The massive penetration of EV's into modern transport sector calls for proper regulation and coordination mechanisms in the xEVs charging behaviors. Numerous proposals had been suggested in this aspect. In [17], the notion of time coordinated charging (TCC) and power coordinated charging (PCC) is proposed. Designing a globally optimal scheduling scheme is impractical because future information about the arrival of vehicles is not available, consequently is hard to estimate the upcoming load. However, authors in [18] attempts to formulate a realtime pricing model based optimization problem for global scheduling of PHEV charging and discharging. In the same paper they also proposed a scalable local scheduling optimization problem which can achieve the performance close to that of global counterpart. A real-time approach to TCC is introduced in [9] and similarly real time PCC is addressed in [10] to minimize the power attenuation and shaping voltage profile during PHEV charging process. Request from vehicle or users are processed in [19] and [20] to devise hierarchical aggregator-based hybrid vehicles charging control mechanisms that may lower the variability of demand and distributed power generation through smart charging and discharging in real time. Furthermore, [9] also classifies PHEV charging strategies into architecture based as well as control based. The former may be designed either in centralized or decentralized mode while there may be direct or incentive based control in the latter. A game theoretic paradigm is adopted in [9] to supervise the random crowd of vehicle fleet thereby safeguarding PHEV's privacy issues in terms of charging schedules. In [10], a fuzzy logic Fuzzy Charging Controller (FCC) is proposed that takes the States of Charge (SOC), the grid parameters represented in the system minimum voltage, and the hourly energy price as input parameters to keep the system threshold voltage within permissible limits. The interpolated FFT algorithm employed by authors of [7] attempts to devise an cost effective interactive charging strategy which maintains grid stability by minimizing the grid load factor, and ceils the optimal battery charging rate within admissible limits. The work in [11] demonstrates the optimization of charging cost of PHEV fleet through moving window optimization scheme. The authors in [21] adopted a dynamic programming approach to simulate a bidirectional control flow model (V2G also enabled) that coordinates the charging scheme.

A master coordination framework having reliable service oriented software architecture (SOA) should thus be developed that provide a platform for the XEV users which enable them to participate in regular and auxiliary energy market operations [22]. Such platform is analogous to power exchanges (PXs) [23]. It feds the daily demand and supply trends on its database and correspondingly settles a proper market clearing price for the involved stakeholders such that the interest of each players is respected in a win-win paradigm. Figure 1 shows the snapshot of one day market clearing volume corresponding to stipulated clearing price. One day market clearing scenario as shown in Fig. 1 was obtained from the historical data of Indian Energy Exchange to show the market price and cleared volume variability in Indian scenario. In Fig. 1, abscissa represents the time

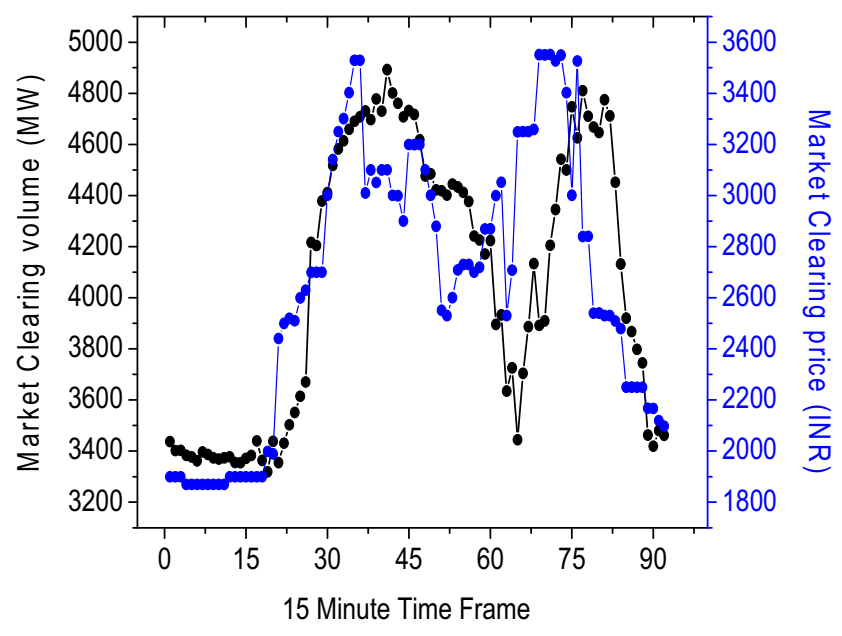

Fig. 1 Load and price curve for a typical power market 
interval and ordinate represent market clearing volume (MW) and market clearing price (INR). The detail study of Fig. 1 represents how the price and volume depends on time horizon throughout the day. The observation of graph reveals the two major conclusions, first is the morning and evening time horizon the demand of energy is high and second is price is proportional to the varying demand. Hence, the night horizon time quantum would be more suitable for home charging system and the day based lower price and demand time horizon will be more suitable for public EVs charging stations. Cloud computing has recently emerged as technology enabler for the smart cities, smart health, smart transportation, and smart environment as well as for smart gird [18].

Through the implementation of Cloud monitoring and management the customers can access the required data at anytime and anywhere. Cloud enables user to remotely available, generating a huge cost-savings-especially on offerings that require frequent updates to stay competitive. The added flexibility enables EV users to quickly and easily scale according to demand. Connectivity enables efficient and effective maintenance and support of each charger in the field. At the same time, connectivity also allows $\mathrm{EV}$ users to monitor both the development of the market and customer requirements. The EV charging market is rapidly evolving and so is the evolution of customers' needs. Cloud computing utilities provide massive virtualized storage spaces which can be deployed in pay-per-use models to realize warehouse or database needs of such software. Contemporary developments in the intelligent transportation systems (ITS) aided with utilities for information and communication technologies (ICT) have enabled bidirectional data collection and transport mechanics where the real-time vehicle data can be utilized for modeling fleet charging management prototypes [24].

Motivated by the facets of cloud computing paradigms and the need for an efficient frame work for coordinated charging of $\mathrm{xEVs}$, this work proposes centralized cloud based remote xEV charging management system. Figure 2 depicts the integrated xEV data aware fleet management architecture constituted by data and energy trade mechanisms among the acting entities. Inheriting the ideology of duplex power and data exchange services, the work demonstrates a prototyped software that feds XEV customer parameters such as current geographical location, state of charge of xEV battery etc, and correspondingly guides him to appropriate charging station.

The application acts analogous to an energy exchange (EX) that accumulates the bids from multiple vendors and aggregators and at the same instant extracts the day ahead or term ahead demand market attributes, for realizing a customer centric recommendation environment. The work also presents the software specifications required to prototype similar applications.
The detailed contributions are summarized as follows:

1. Proposed a cloud based remote xEV charging management framework recommending optimal charging solution to the involved $\mathrm{xEV}$ customers.

2. Proposed a prototypical software description for developing such applications.

3. The ER diagram, use case diagram and dataflow diagram for a typical $\mathrm{xEV}$ charge management software is developed that will support a consistent standard for the programmers and application developers.

4. Highlighted the cyber threats that may arise while installing such applications and also addressed the need of security and integrity enforcements while running such applications.

The manuscript is organized as follows. "Business Model" explains operational business model for the stakeholder entities. "Software Description of the Proposed System" describes the software description for developing prototypical smart charge management system. In "Proposed System Output Parameters" the functional aspects of the developed system is outlined along with the cyber issues that may arise in course of application execution. Three case studies are presented in "Case Studies" followed by the conclusion in "Conclusion".

\section{Business Model}

This proposed system xEVs Charging Management System demonstrate the significant of cloud based paradigm

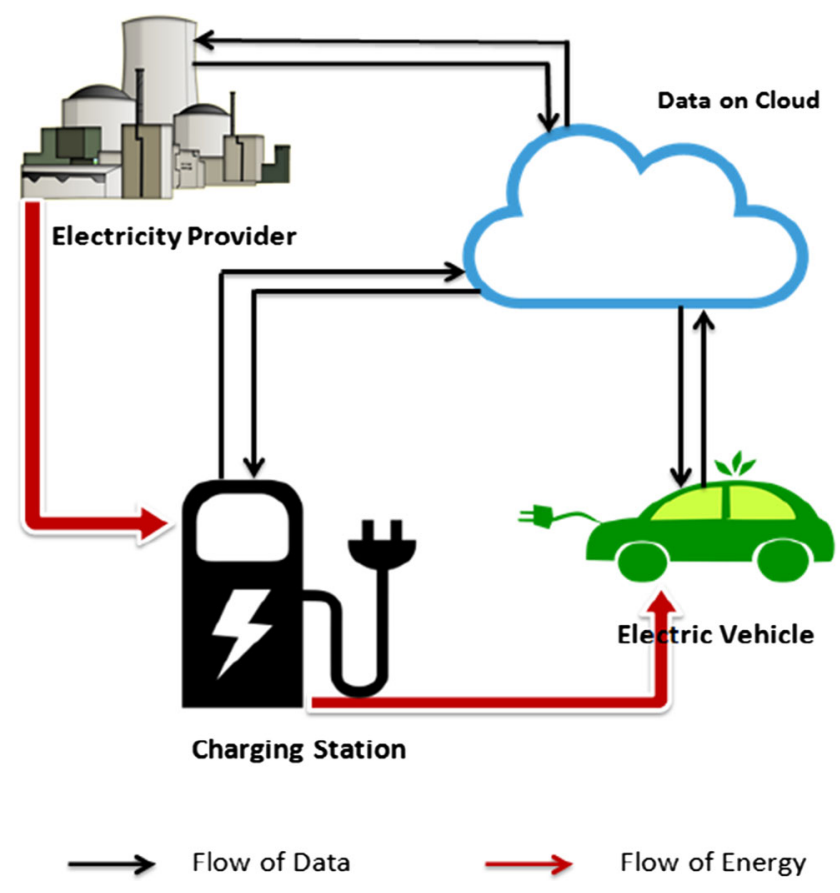

Fig. 2 Data and energy flow for xEVs charging Management 
focused to deploy a smart and coordinated charging management framework for XEV fleet. The front End of the software is developed using ASP.NET to have better GUI realization for system end, while for backend operations, C\#.NET is used.

An instance of data center is developed using SQL Server 2008 purposed to manage the computation and analytics tasks such as login (authentication), searching, updating information regarding energy attributes and bidding analysis. The proposed system demonstrate the Bid and auction mechanism under took by Power system stack holders. It exposes a virtual market place for the electric vehicle users who track the real time energy market attribute such as present power rate predicted power flow and cost. The cloud analytics undergoing at the data center employees computationally perceptive be data analytics to recommend smart charging options to the corresponding vehicles customer. The application supports dedicated interfaces to provide real time information to the $\mathrm{xEV}$ user regarding nearest charging station that can provide energy at economy rate and that so by ensuring a minimum queuing delay (Fig. 3).

The application acts not only as a recommendation engine for the xEVs Users but also provide an optimal charging solution to all entities involved in a smart charging infrastructure such as charging station, aggregators, smart grid etc.
Charging stations manage their user through this application performing task such as updating charging level, places to stay, update list of selected consumer after bidding etc. This application reduce load on smart grid by solving the problem of demand and supply problem.

\section{Software Description of the Proposed System}

This section presents the detailed software description of the prototype system using different models for representing interaction among entities, the schema description, the control and work flow among the involved actors etc.

\section{ER-Diagram}

An entity relationship diagram (ERD) represents the relationships among entity sets stored in the database. An entity in this context is a component of data. In other way to say, entity relationship diagrams illustrate the logical configuration of databases. There are three main entities involved in the model:-

1) Admin: The role of "Admin" will perform by any centralized unit/Power System Operator (PSO) to control the available as well as new constructed charging
Fig. 3 Schematic diagram of the business model

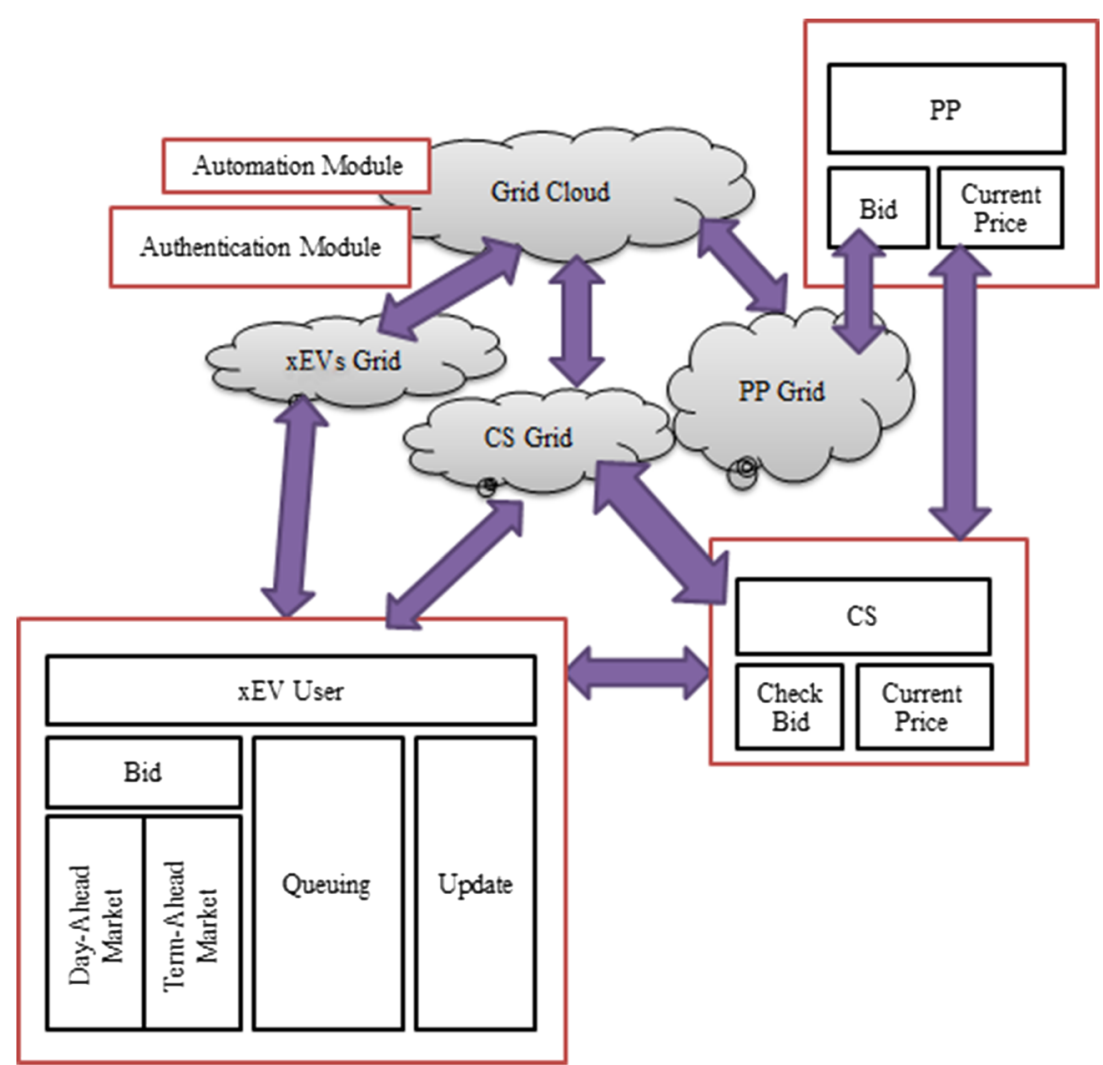


station and power plants. This unit would be organized by government or any private firm. Power plants and Charging stations have to forward all the necessary information along with documents and then the PSO will verify and will upload all the related meaningful information on cloud. It may be Power. The PSO (admin) entity has two major properties "Admin ID" and "Password" to login in cloud data system and perform all other related task (i.e. Fig. 4). For the proposed application the administrative legacy will be licensed to different stakeholders based on the registration and legal procedures.
2) Power Plant Operator: The power plant operator (PPO) or independent system operator (ISO) registered by admin with a unique PP_ID and a self-assigned password which can later be changed by Power Plant operator. Further attributes of Power Plant Operator are name, location (updatable), contact details (address, email, phone no.) and energy transformation related (current price, working/transformation time, capacity etc.).

3) Charging Station Operator: The Charging Station Operator (CSO) also registered by Admin with a unique CS_ID and a self-given Password which can later change
Fig. 4 ER-Diagram of xEVs charging Management

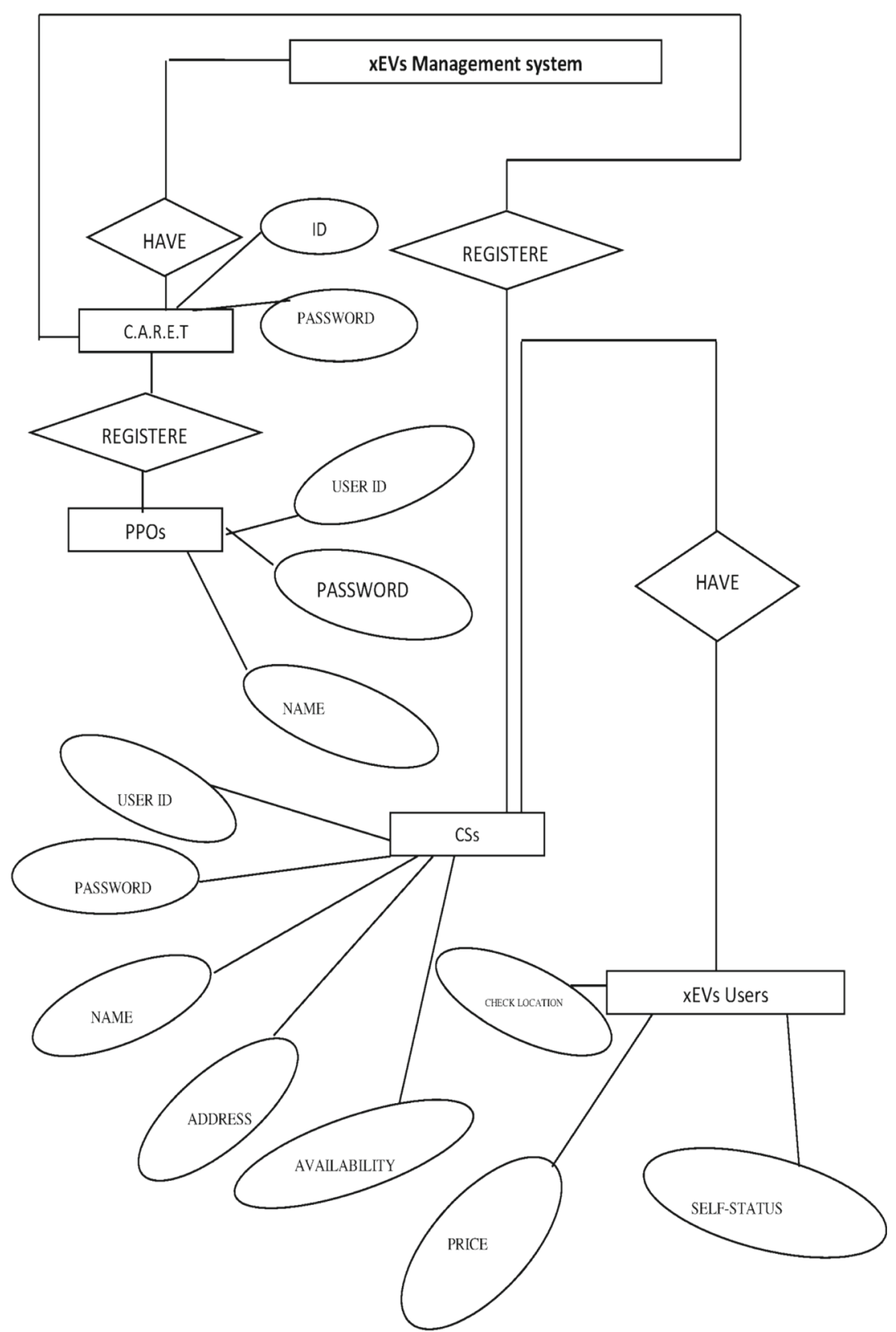


by Charging Station Operator. Further properties of Charging Station Operator are name, location, contact details (address, email, phone no.), energy transformation related (current price, working/transformation time, capacity etc.) and a record of nearby places where xEVs user can stay.

4) xEVs User: The $x E V$ user can register themselves using an online form filling process. After admin approval its session is assigned through a unique USER_ID.

$\mathrm{xEV}$ users have further properties like name, contact details (address, email and phone no.), xEV vehicle identification number (VIN), and type of xEV battery etc.

\section{Use Case Diagram}

Use case diagrams also referred to as behavior diagrams, are used to describe a set of actions (use cases) that some system or a batch of systems (subject) will or can perform in collaboration with one or more external users of the system (actors). In other words, it represent to which functionality (s) the actors/entities in the system involved for. Each use case should provide some observable and valuable result to the actors or other stakeholders of the system. For the proposed framework, in correspondence with $\mathrm{xEV}$ user, the application will utilize the key attributes such as GPS location of $\mathrm{xEVs}$, the state of charge (SOC) and state of health $(\mathrm{SOH})$ of $\mathrm{xEV}$ battery and other miscellaneous attributes to calculate the electric vehicle range anxiety (EVRA) in percentage as shown in Fig. 5.

a) The application will be able to track status or level of charging (SOC) of their own XEV through the application.

b) Booking and bidding for the energy at minimum tariff.

c) It can identify location of optimal charging station, as the system will recommend a set of options in decreasing order of priority.

The Charging Station Operator (CSO) is the second level user who gets control over the consumers and self-database and also has seamless communication with smart grid operators followed by authentication from the admin. This entity can perform the tasks such as:

a) Control over self-database such as update price for consumer, update demand for main suppliers etc.

b) Management for the xEVs such as give notification when EV charged, give availability etc.

c) Fix the lowest price for bidding and final the price to buy from main supplier and sell to the consumer.

Power Plants benefits operator is the main supplier of energy who has various tasks after login such as

a) View data of CSs such as consumers, average expenditure, location etc. b) Management of self-records such as update energy price, availability of energy etc.

c) View and sell energy after Bidding.

\section{Data Flow Diagram [25]}

The data flow diagram (DFD) illustrates the graphical depiction of the flow of data among the entities of software. In structural design of software modeling, a DFD can visualize the data processing steps. At the $\mathrm{xEV}$ user end, the application will take state of charge (SOC) and state of health $(\mathrm{SOH})$ of $\mathrm{xEV}$ battery, the current geographical coordinates of the $\mathrm{xEV}$, and the degree of EVRA etc. as input and provides the optimal location of the charging station as recommended output. Further, when the user is a charging station vendor, the application will feed the bidding volume,

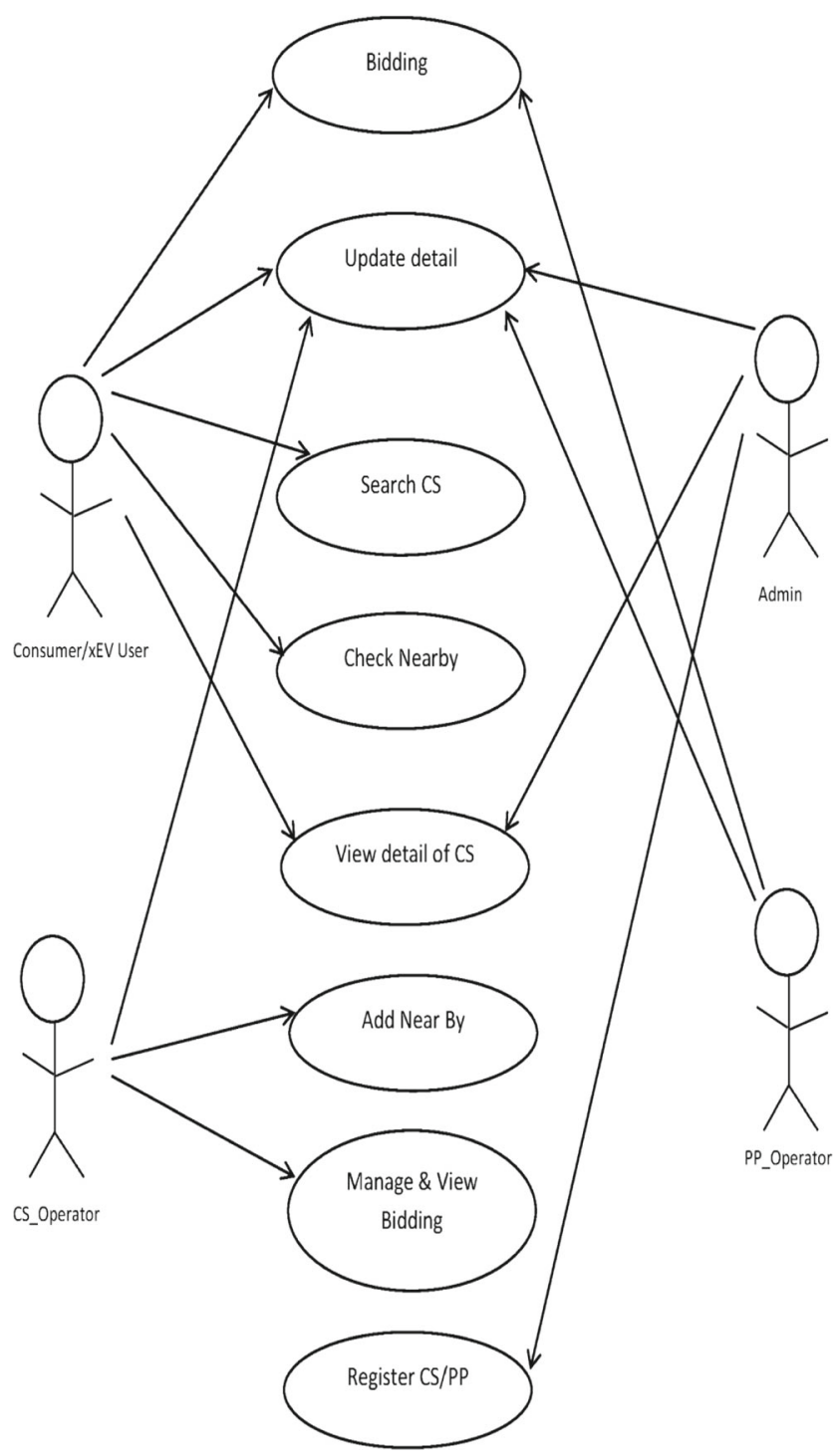

Fig. 5 Use case diagram for proposed model 
bidding price and the geo-distribution of $\mathrm{xEVs}$ in the vicinity etc., as input and engages customers so as to maximize its profit (Fig. 6).

\section{Proposed System Output Parameters}

The application can be deployed in various operating environments such as smart phones, charging station outlets, servers etc. Based on the portability needs of the execution platform or operating system the application can be augmented with compatible updates or programming reorganization [26]. However, the software will act as a standard to open doorway to software developers to program applications dedicated to smart grid utilities and services. Figure 7 shows a snapshot of the pilot application while running at the $\mathrm{xEV}$ user end.

Moreover, the framework may also be prone to the cyber threats thus causing in cyber-physical threats for the overall smart grid ecosystem. Since the underlying smart grid system that is carrying the $\mathrm{xEV}$ fleet is a complex system, comprising of massive hardware components, requires pervasive control and monitoring infrastructures. The cloud aware technologies play fundamental role underpinning varying smart grid functionalities, to automate remote management tasks such as xEV fleet management, perform event driven processes etc. Such intensive use of cyberphysical infrastructure poses serious threats with respect to privacy, authentication, intrusion and novelty of such framework [27]. Any breach in such a delicate system from cyber fabric may have serious consequences such as power interrupt, chaos in the fleet due to mismanagement, data breach, accidents etc. Therefore, a holistic and secure cyber framework need to be deployed to address the possible vulnerabilities presented in varying modes of execution of the application.

\section{Case Studies}

Three case studies are presented here namely Electric Vehicle Managed Charging Demand Response, Current demand for electric vehicles, Aggregate Demand Supply System

\section{Electric Vehicle Managed Charging Demand Response}

According to [28], V2G has been tested in a small number of pilots, a number of technical and regulatory issues need to be resolved before it can be widely and effectively used. While managed charging also faces some barriers, solutions are in process and could help prepare a solid foundation for $\mathrm{V} 2 \mathrm{G}$. Using managed charging as an effective grid resource-with benefits for customers and utilities - could represent a compelling opportunity for utilities. As of February 2017, more than 580,000 EVs were sold in the United States, 2 representing approximately one terawatt-hour (TWh) of annual consumption.3 According to Bloomberg New Energy Finance (BNEF), EV electricity consumption is projected to increase to approximately 33 TWh annually by 2025 , and 551 TWh by 2040.4 Given the projected growth in EVs, and the increasing need for flexible grid resources, more utilities are evaluating the

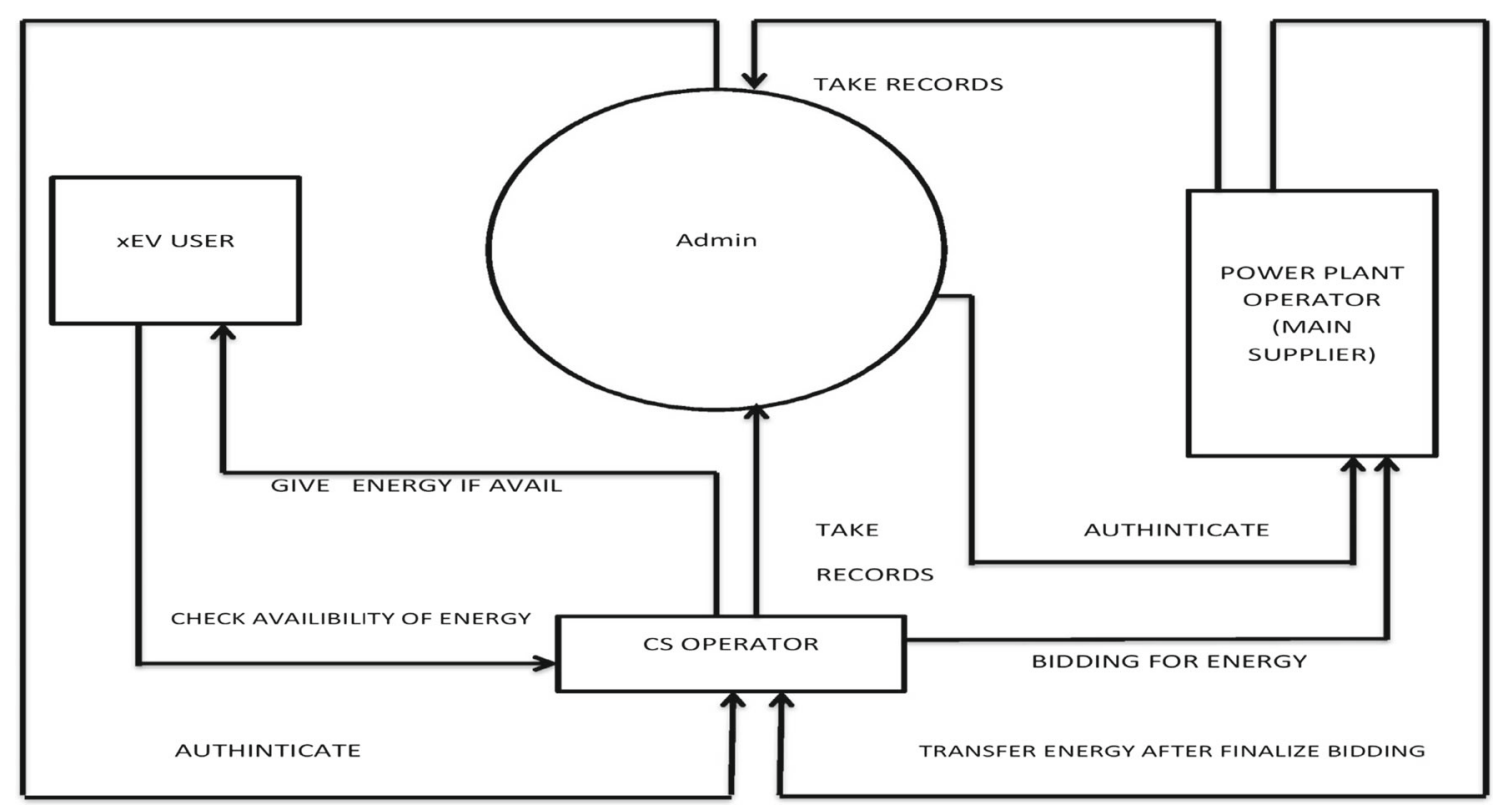

Fig. 6 DFD for the proposed model 


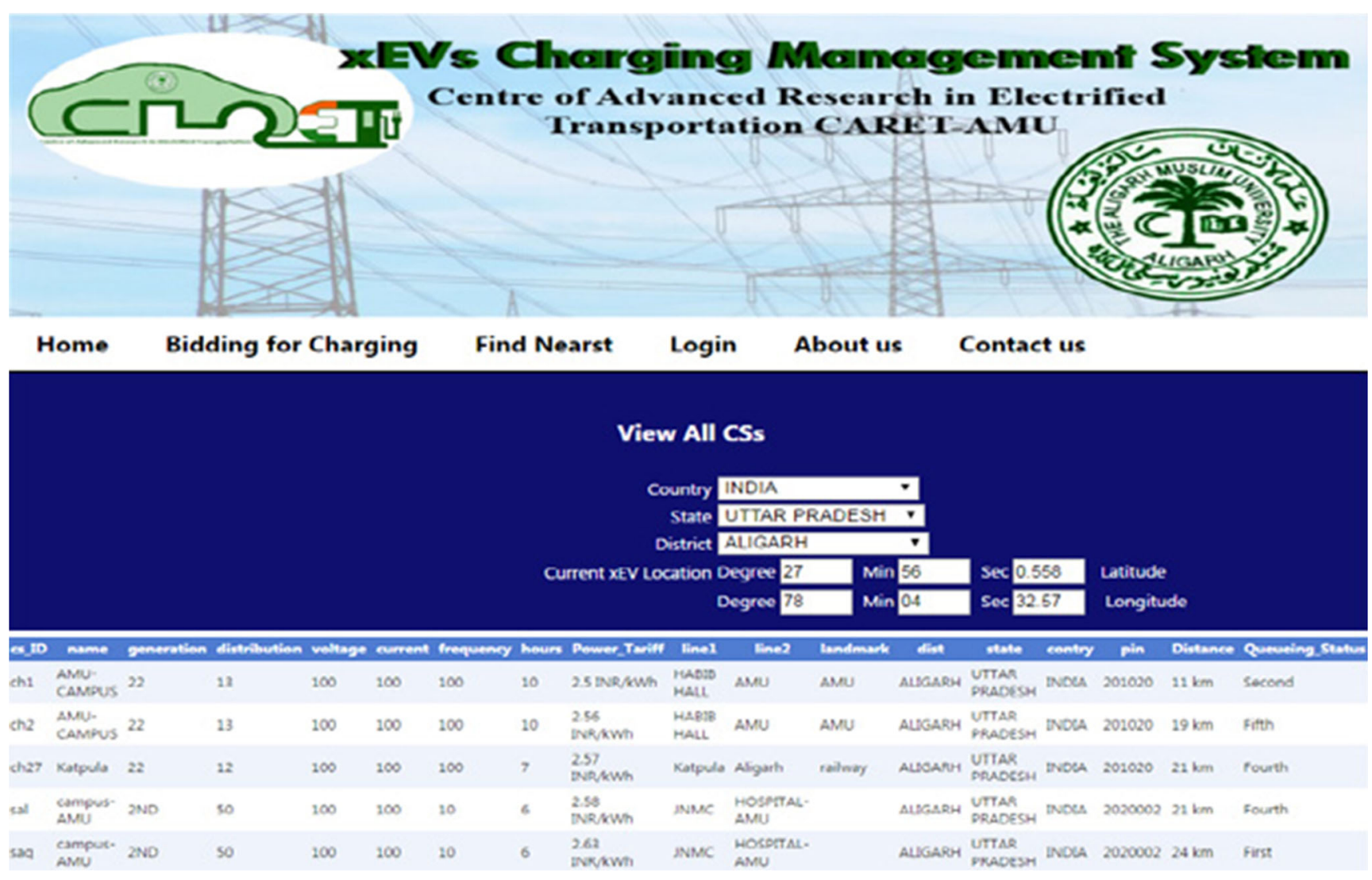

Fig. 7 Sample output for the proposed application

opportunity for managed charging. In the Smart Electric Power Alliance's (SEPA) 2017 Utility Demand Response Survey, 69 percent of respondents indicated that they are planning, researching, or considering DR programs that integrate EV managed charging, compared to 20 percent that, at present, have no interest (Fig. 8).

\section{Current Demand for Electric Vehicles}

According to [29], Indian demand in the overall EV market is sluggish. High upfront costs and service anxiety

Electric Vehicle Managed Charging Demand Response

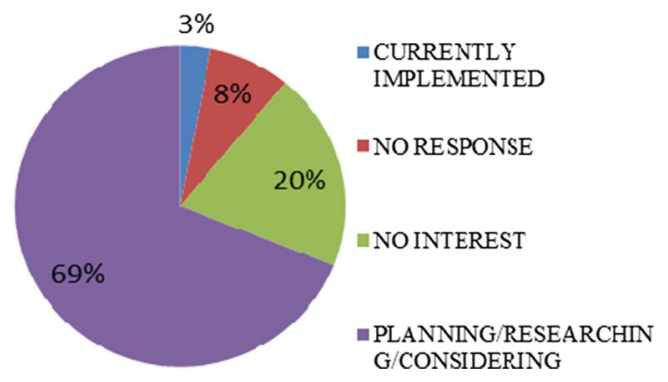

Fig. 8 Electric vehicle managed charging demand response [28] around re-charging/maintenance facilities have been barriers to sales growth. Only 130,000 hybrid and electric vehicles have been sold in 2012, according to media reports. The market is dominated by two wheelers, with 97-98\% of sales occurring for electric bikes, scooters and motorbikes. In the $4 \mathrm{~W}$ market, the financial case for investing in HEVs/EVs is weak. The costs of energy storage technology need to decrease so that EVs can financially compete with fuel efficient 'normal' vehicles. The National Electric

\section{Total Reported EV Sales in India}

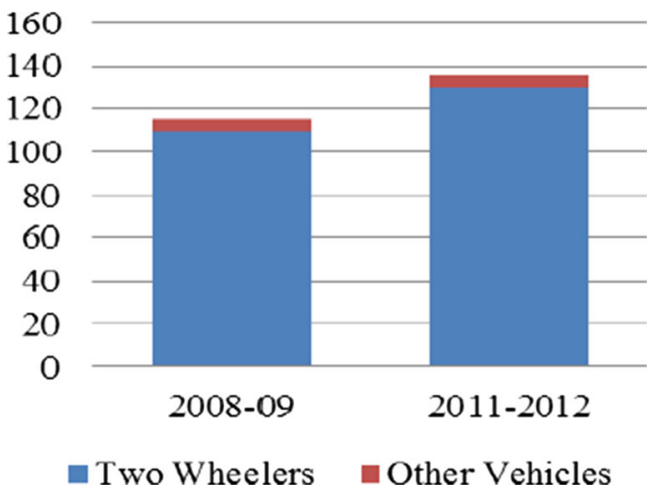

Fig. 9 Sluggish EV sales in India 2008-2012 [29] 
Fig. 10 Aggregate Demand Supply System [30]

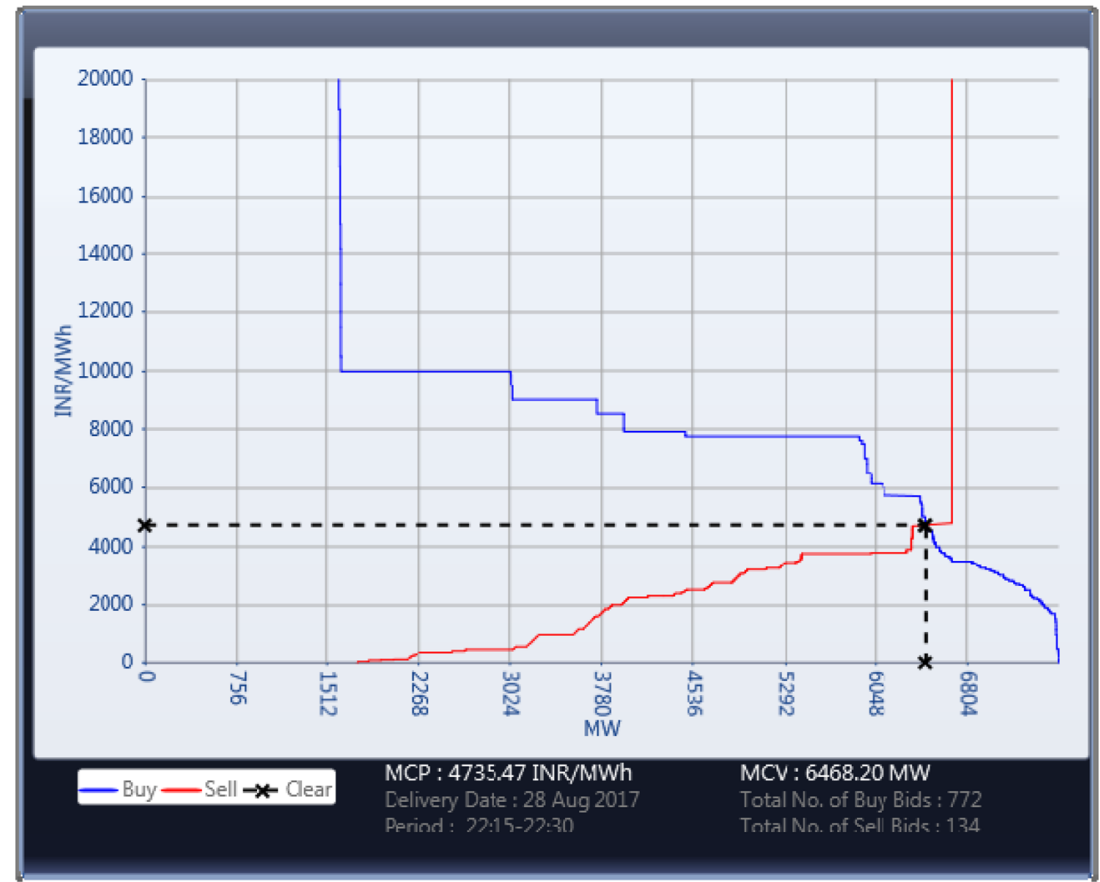

Mobility Mission Plan (NEMMP) target, of 5-7 million EVs by 2020 , corresponds to penetration of EVs of $15 \%$ in $2 \mathrm{~W}$ and $19 \%$ in passenger car markets. This is ambitious, since sales have only grown at $\sim 6 \%$ pa over $2008-12$. For the largest segment, two wheelers, hybrid/EV penetration was estimated to be $1-2$ of total $2 \mathrm{~W}$ sales. Thus, all the yearly trading information from one location to another, consumer behavior, xEVs aggregator services will be executed by the historical data saved at the cloud system, which will help to enhance the deployment of xEVs in India. The deployed EVs infrastructure is shown in Fig. 9.

\section{Aggregate Demand Supply System}

In Fig. 10, chart showing one day (28th Aug, 2017) market scenario (Buy \& Sell). Total No. of buy bids on 28th Aug, 2017 is 773 MW and Total No. of Sell bids is 134 MW, The all the informative data such as no of bidder, seller, cleared volume, market clearing price, locations to withdraw and inject the bided power, and the detail of the costumer behavior based previous traded transactions will be stored on the cloud system in order to execute the proper day ahead as well as term ahead energy trading mechanism.

On the basis performed three studies we found that the reported sales of EVs are very high as shown in Fig. 9 and it also reveals that the demand-response is not considerable which enable the need of robust cloud system as proposed in this manuscript, which make a user enable to get real time response.

\section{Conclusion}

The proposed work presents a cloud aware smart charge management framework for intelligently charging the $\mathrm{xEV}$ fleet. The application provides stakeholder specific interfaces and modules to enable an uninterrupted power flow across the whole smart grid infrastructure and simultaneously circumventing the demand peaks created by the $\mathrm{xEV}$ penetration. When installed at the $\mathrm{xEV}$ customer end, the cloud monitored software will be able to track the geo-distribution of optimal charging station that makes the multi-objectives met concurrently. Among the list of recommending parameters, the application will specific focus on the three requirements in decreasing order or priority namely minimum charging tariff, nearest charging spot and the station having minimum xEV congestion. Moreover, the system will also support dedicated services for charging vendors, aggregators and other market players. The application will track the status of day-ahead and auxiliary market to settle effective bidding and market clearing environment that maintains a win-win strategy for every stakeholders. The work also addresses the need of cyber-physical enforcements to ensure hassle free operation complete SG infrastructure.

Acknowledgements This research is supported by the Centre of Advanced Research in Electrified Transportation (CARET), Aligarh Muslim University, India sponsored by the grant from the Ministry of Heavy Industries, Govt. of India under FAME Mission. 


\section{References}

1. Ahmad F, Alam MS (2017) Feasibility study, design and implementation of smart polygeneration microgrid at AMU. Sustain Cities Soc

2. International Energy Agency (2016) Energy and air pollution. World Energy Outlook - Spec Rep, p 266

3. Guðnadóttir PB Electric vehicles, pp 1-22

4. Pand H (2015) Catch 22: electric vehicles and the required infrastructure

5. Fang X, Misra S, Xue G, Yang D (2012) Smart grid - the new and improved power grid: a survey. IEEE Commun Surv Tutor 14(4):944-980

6. Hannan MA, Azidin FA, Mohamed A (2014) Hybrid electric vehicles and their challenges: a review. Renew Sust Energ Rev 29:135-150

7. Bae S, Kwasinski A (2012) Spatial and temporal model of electric vehicle charging demand. IEEE Trans on Smart Grid 3(1):394 403

8. Asaad M, Ahmad F, Alam MS, Rafat Y (2017) IoT enabled electric vehicle's battery monitoring system. EAI, SGIOT, no 8

9. Xu J, Wong VWS (2011) An approximate dynamic programming approach for coordinated charging control at vehicle-to-grid aggregator. In: 2011 IEEE International Conference on Smart Grid Communications (SmartGridComm), Brussels, pp 279-284. https://doi.org/10.1109/SmartGridComm.2011.6102333

10. Alam MS (2013) Key barriers to the profitable commercialization of plug-in hybrid and electric vehicles. Adv Automob Eng 2(2):1-2. https://doi.org/10.4172/21677670.1000e117

11. Bayram IS, Michailidis G, Papapanagiotou I, Devetsikiotis M (2013) Decentralized control of electric vehicles in a network of fast charging stations. In: Globecom 2013-symposium selected areas in communications (GC13 SAC), pp 2785-2790

12. Wu C, Member S, Mohsenian-rad H (2012) Vehicle-to-aggregator interaction game. IEEE Trans on Smart Grid 1(3):434-442. https://doi.org/10.1109/TSG.2011.2166414

13. Wang Q, Liu X, Du J, Kong F (2016) Smart charging for electric vehicles: a survey from the algorithmic perspective. IEEE Commun Surv Tutor 18(2):1500-1517

14. Chan CC, Jian L, Tu D (2014) Smart charging of electric vehicles-integration of energy and information. IET Electr Syst Transp 4(4):89-96

15. Kang Q, Member S, Wang J, Zhou M, Ammari AC (2016) Centralized charging strategy and scheduling algorithm for electric vehicles under a battery swapping scenario. IEEE Trans Intell Transp Syst 17(3):659-669. https://doi.org/10.1109/TITS. 2015.2487323

16. Eurelectric (2015) Smart charging: steering the charge, driving the change. no. March, p 57

17. Abaker I et al (2015) The rise of 'big data' on cloud computing: review and open research issues. Inf Syst 47:98-115

18. Aburukba R (2015) Role of internet of things in the smart grid technology. no. May, pp 229-233

19. Alonso M, Amaris H, Germain JG, Galan JM (2014) Optimal Charging Scheduling of Electric Vehicles in Smart Grids by Heuristic Algorithms. Energies 7(4):2449-2475. https://doi.org/10. 3390/en7042449

20. Awaad MH, Krauss H, Schmatz HD (1978) Advanced praise for the unified modeling language reference manual, 2nd edn, vol 240(3)

21. Scaife N, Carter H, Traynor P, Butler KRB (2016) CryptoLock (and drop it): stopping ransomware attacks on user data. In: 2016 IEEE 36th International Conference on Distributed Computing Systems (ICDCS), Nara, pp. 303-312. https://doi.org/10.1109/ICDCS.2016.46

22. Yang Y, Wu L, Yang S (2012) The structure of intelligent grid based on cloud computing and risk analysis. In: 2012 4th international conference on intelligent human-machine systems and cybernetics IHMSC 2012, vol 2, pp 123-126

23. Khan Q, Ahmad F, Imran M (2017) Congestion management in Indian Power Transmission System. Int J Eng Technol 9(3):2631

24. Alam MS Vehicle to Cloud(V2C) remote management of Electric, Hybrid and plug-in Hybrid Electric Vehicle Charging.pdf. p. Patent Application \# 34590/DEL/2015

25. Elbakush A Functional modeling with data flow diagrams, Utrecht University. Available at: http://www.students.science.uu. nl/ 5771668/me/AssignmentD_5771668_Elbakush.pdf

26. Cyber-Physical systems_from theory to practice. CRC Press (2015).pdf

27. Sharif H A survey on cyber security for smart grid a survey on cyber security for smart grid communications, no. August 2014

28. THEC For and M Charging (2017) utilities and electric vehicles the case for. no. April

29. Market Analysis_The case for electric and hybrid vehicles in India_sustainabilityoutlook

30. Demand Supply Curves_Indian Energy Exchange Ltd 